\title{
INVOLUTIONS ON ALGEBRAS ARISING FROM LOCALLY COMPACT GROUPS
}

\author{
PAUL L. PATTERSON
}

(Communicated by Dale Alspach)

\begin{abstract}
Two Banach algebras are naturally associated with a locally compact group $G$ : the group algebra, $L^{1}(G)$, and the measure algebra, $M(G)$. For these two Banach algebras we determine all isometric involutions. Each of these Banach algebras has a natural involution. We will show that an isometric involution, $\left({ }^{\#}\right)$, is the natural involution on $L^{1}(G)$ if and only if the closure in the strict topology of the convex hull of the norm one unitaries in $M(G)$ is equal to the unit ball of $M(G)$.

There is a well-known relationship between the involutive representation theory of $L^{1}(G)$, with the natural involution, and the representation theory of $G$. We develop a similar theory for the other isometric involutions on $L^{1}(G)$. The main result is: if $\left(^{*}\right)$ is an isometric involution on $L^{1}(G)$ and $T$ is an involutive representation of $\left(L^{1}(G),{ }^{\#}\right)$, then $T$ is also an involutive representation of $L^{1}(G)$ with the natural involution.
\end{abstract}

\section{INTRODUCTION AND NOTATION}

In the study of locally compact groups two group algebras are of special interest. In 1952 Wendel showed that the algebra $L^{1}(G)$, of all absolutely integrable functions on $G$ considered as a Banach algebra (with convolution multiplication), characterizes the locally compact group $G$ up to homeomorphic isomorphism [10]. In 1964 Johnson using Wendel's result proved that $M(G)$, the algebra of complex regular Borel measures on $G$ (with convolution multiplication and the total variation norm), is also a complete set of invariants for $G$ [6].

Both of these group algebras have a natural involution, but the involution was not used by either Wendel or Johnson. Although the natural involution on $L^{1}(G)$ was not used by Wendel, it is of importance in the representation theory of locally compact groups. In [8] we used the involutive structure to give characterizations of $L^{1}(G)$ and $M(G)$ as Banach *-algebras. The question arises, how unique are the natural involutions on $L^{1}(G)$ and $M(G)$ ? Using some of the results of [8] we will show that for most groups the natural involution on either $L^{1}(G)$ or $M(G)$ is far from being unique.

Received by the editors July 21,1992 and, in revised form, October 6, 1992.

1991 Mathematics Subject Classification. Primary 43A20; Secondary 43A10, 46K99.

Key words and phrases. Locally compact groups, Banach *-algebras, isometric involutions. 
In Theorem 1.2 we will determine all isometric involutions on $L^{1}(G)$. All isometric involutions, $\left(^{\#}\right)$, on $L^{1}(G)$ are of the form $f^{\#}=\chi \cdot\left(f^{*} \circ \sigma\right)$, where $\chi$ (a character on $G$ ) and $\sigma$ (an automorphism of $G$ ) satisfy certain conditions, and $\left(^{*}\right)$ is the natural involution on $L^{1}(G)$. Since $M(G)$ is the double centralizer algebra of $L^{1}(G)$, there is a one-to-one correspondence between isometric involutions on $L^{1}(G)$ and isometric involutions on $M(G)$; this allows us to determine all the isometric involutions on $M(G)$.

There is a condition on the algebraic and analytic structure of $L^{1}(G)$ which differentiates the natural involution. If $\left(^{\#}\right)$ is an isometric involution on $L^{1}(G)$ and $S$ is the set of norm one unitaries in $M(G)$ (with respect to the involution induced from $\left.\left(L^{1}(G),{ }^{\#}\right)\right)$, then $\left(^{\#}\right)$ is the natural involution if and only if the unit ball in $M(G)$ is equal to the closure, in the strict topology induced by $L^{1}(G)$, of the convex hull of $S$.

The representation theory of $L^{1}(G)$, with the various involutions, also shows that the natural involution is of particular importance. If $\left(^{\#}\right)$ is an isometric involution on $L^{1}(G)$ and $\left(^{*}\right)$ is the natural involution, then any *-representation of $\left(L^{1}(G),{ }^{\#}\right)$ is also a ${ }^{*}$-representation of $\left(L^{1}(G),{ }^{*}\right)$. This fact and the characterization of isometric involutions allow us to determine which of the positive definite functions give rise to the positive functionals of $\left(L^{1}(G)\right.$, \#). With the natural involution, $L^{1}(G)$ is a ${ }^{*}$-semisimple Banach ${ }^{*}$-algebra. In contrast, for every open normal subgroup, $N$, of index two there is an involution, $\left({ }^{\#}\right)$, associated to $N$ such that $\left(L^{1}(G),{ }^{\#}\right)$ is a \#-radical algebra . Lastly we will discuss the relationship between the nondegenerate \#-representations of $\left(L^{1}(G),{ }^{\#}\right)$ and the continuous representations of $G$.

Notation. In $\S 1$ we will use the notation developed in [8]. But in the interest of clarity, we define the following two topologies. If $I$ is a two-sided ideal in a normed algebra $B$, then the strict topology on $B$ is the topology induced by the family of psuedonorms $\|\cdot\|_{b}, b\|\cdot\|$ for $b \in I$, where

$$
\|a\|_{b}=\|a b\| \text { and }{ }_{b}\|a\|=\|b a\| \text { for all } a \in B \text {. }
$$

We denote the strict topology on $B$ with respect to $I$ by $S(B, I)$. If $A$ is a Banach space and $V$ is a subset of $A^{*}, \sigma(A, V)$ will denote the weak topology on $A$ induced by $V\left[8\right.$, p. 491]. Thoughout this paper we will use $\left(^{*}\right)$ to denote the natural involution on $L^{1}(G) ; f^{*}(u)=\Delta\left(u^{-1}\right) f\left(u^{-1}\right)^{-}$for $f \in L^{1}(G)$. For representation theory we will use the notation developed in $[3,4]$.

\section{ISOMETRIC INVOLUTIONS ON $L^{1}(G)$ AND $M(G)$}

Thoughout this section $G$ will denote a locally compact group. We will use the following lemma in our characterization of the isometric involutions on $L^{1}(G)$. The proof, which follows from the fact that since $G$ is completely regular, $C_{c}(G)$ separates points in $G$, is left to the reader.

Lemma 1.1. Let $G$ be a locally compact group, $\tau$ a homeomorphism of $G$ onto $G$, and $g$ a continuous map from $G$ into $\mathbb{C}$. If for all $f \in L^{1}(G)$ we have $f(\tau(u))=g(u) f(u) \lambda$-a.e., then $\tau$ is equal to the identity homomorphism and $g(u)=1$ for all $u \in G$.

Theorem 1.2. Let $G$ be a locally compact group, and let $\left(^{\#}\right)$ be an isometric involution on $L^{1}(G)$. Then there exists a unique pair, $(\sigma, \chi)$, of a homeomorphic 
automorphism, $\sigma$, of $G$ and continuous group homomorphism, $\chi: G \mapsto \mathbb{T}$, such that

(1) $\sigma^{2}$ is equal to the identity (hence $\sigma$ also satisfies $\lambda_{G} \circ \sigma=\lambda_{G}$ and $\left.\Delta_{G} \circ \sigma=\Delta_{G}\right)$,

(2) $\chi \circ \sigma$ equals $\chi^{-}$and

$$
f^{\#}(u)=\chi(u)\left(f^{*} \circ \sigma\right)(u)=\chi(u) \Delta\left(u^{-1}\right) f\left(\sigma(u)^{-1}\right)^{-} .
$$

Furthermore, if the pair $(\sigma, \chi)$ satisfies these conditions, then the last expression defines an isometric involution on $L^{1}(G)$.

Proof. Let $\lambda$ equal $\lambda_{G}$ and $\Delta$ equal $\Delta_{G}$. Define $\theta: L^{1}(G) \mapsto L^{1}(G)$ by $\theta(f)=f^{* *}$. Since $\theta$ is an isometric automorphism of $L^{1}(G)$, [10, Theorem 5] implies there exist $\chi^{\prime}, \sigma$, and $c$ with $\theta(f)(u)=c \chi^{\prime}(\sigma(u)) f(\sigma(u)) \lambda$-a.e., where $\chi^{\prime}$ is a continuous homomorphism of $G$ into $\mathbb{T}, \sigma$ a homeomorphic automorphism of $G$, and $c$ a positive number such that $\lambda(\sigma(V))=c \lambda(V)$ for each open set $V \subset G$. Set $\chi$ equal to $\chi^{\prime} \circ \sigma$. A calculation shows that $f^{*}(u)=(\theta(f))^{*}(u)=c \chi(u) \Delta\left(u^{-1}\right) \Delta(\sigma(u)) f^{*}(\sigma(u)) \lambda$-a.e. If we apply the above expression to $f^{\#}$, we have

$$
f(u)=\left(f^{\#}\right)^{\#}(u)=c^{2} \chi(u) \Delta\left(u^{-1}\right) \Delta(\sigma(u)) \chi(\sigma(u)) f\left(\sigma^{2}(u)\right) \quad \lambda \text {-a.e. }
$$

By Lemma 1.1 we conclude that $\sigma^{2}$ equals the identity automorphism and that $g(u)=c^{2} \chi(u) \Delta\left(u^{-1}\right) \Delta(\sigma(u)) \chi(\sigma(u))$ is identically one. If $V$ is an open, $\lambda$ finite subset of $G$, then $\lambda(V)=\lambda \circ \sigma^{2}(V)=c^{2} \lambda(V)$; hence $c$ is equal to one. Next $[3,19.17]$ and $\lambda \circ \sigma=\lambda$ imply $\Delta(u) \lambda(V)=\Delta(\sigma(u)) \lambda(V)$, where $u \in G$ and $V$ is an open $\lambda$-finite subset of $G$; thus $\Delta \circ \sigma=\Delta$. Applying this to $g(u)=1$ implies $\chi(u) \chi(\sigma(u))=1$; thus $\chi(u)^{-}=\chi(\sigma(u))$ as claimed. The last statement of the theorem follows from an easy calculation.

Next we will characterize all the isometric involutions on $M(G)$. Johnson showed any isometric isomorphism of $M(G)$ onto $M(G)$ maps $L^{1}(G)$ onto $L^{1}(G)[6]$. Thus if $\left({ }^{\#}\right)$ is an isometric involution on $M(G)$, then $L^{1}(G)$ is a \#-ideal of $M(G)$.

Theorem 1.3. Let $G$ be a locally compact group, and let $\left({ }^{*}\right)$ be an isometric involution on $M(G)$. Then $L^{1}(G)$ is a \#-ideal and there exists a unique pair, $(\sigma, \chi)$, of a homeomorphic automorphism, $\sigma$, of $G$ and continuous group homomorphism, $\chi: G \mapsto \mathbb{T}$, such that

(1) $\sigma^{2}$ is equal to the identity (hence $\sigma$ also satisfies $\lambda_{G} \circ \sigma=\lambda_{G}$ and $\left.\Delta_{G} \circ \sigma=\Delta_{G}\right)$,

(2) $\chi \circ \sigma$ equals $\chi^{-}$and

$$
\left\langle\mu^{\#}, g\right\rangle=\int_{G} g(u) \chi(u) d\left(\mu^{*} \circ \sigma\right)(u)=\left\langle\chi \cdot\left(\mu^{*} \circ \sigma\right), g\right\rangle \quad \text { for } g \in C_{0}(G) \text {. }
$$

Futhermore if the pair $(\sigma, \chi)$ satisfies these conditions, then the last expression defines an isometric involution on $M(G)$.

Proof. Since $M(G)$ contains no annililators of the ideal $L^{1}(G)$, each involution of $L^{1}(G)$ is extendable to at most one involution on $M(G)$. By the above

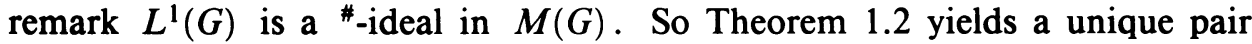
$(\sigma, \chi)$, satisfying (1) and (2), such that $\mu^{\#}=\chi \cdot\left(\mu^{*} \circ \sigma\right)$ for all $\mu$ in $M_{a}(G)$. 
Since $\mu \mapsto \chi \cdot\left(\mu^{*} \circ \sigma\right)$ is an isometric involution on $M(G)$, this identity is valid for every $\mu$ in $M(G)$.

In Theorems 1.2 and 1.3 the natural involution is given by $\sigma$ equal to the identity automorphism and $\chi$ equal to the trivial character. From now on we will refer to all other involutions as the unnatural involutions.

By Wendel [10] the algebraic and analytic structure of $L^{1}(G)$ without the involution determines $G$, which in turn defines the natural involution on $L^{1}(G)$. Hence the algebraic and analytic structure of $L^{1}(G)$ must determine the natural involution. We make this inference explicit with the following theorem. The proof of the theorem uses the following lemma, the proof of which is an easy calculation using Theorem 1.3.

Lemma 1.4. Let $G$ be a locally compact group and (\#) an isometric involution on $L^{1}(G)$. Let $\chi$ and $\sigma$ satisfy the conditions of Theorem 1.2. If $\left(^{\#}\right)$ is the induced involution on $M(G)$, then $\left(\delta_{u}\right)^{\#}=\chi(u) \delta_{\sigma\left(u^{-1}\right)}$ for all $u \in G$.

Theorem 1.5. Let $\left(^{\#)}\right.$ be an isometric involution of $L^{1}(G)$, and let $S$ equal $M(G)_{U} \cap M(G)_{1}$, where $M(G)_{U}$ is defined with respect to the involution on $M(G)$ induced from $\left({ }^{*}\right)$. Then $\left(^{*}\right)$ is the natural involution if and only if the convex hull of $S$ is $S\left(M(G), L^{1}(G)\right)$-dense in $M(G)$.

Proof. Let $S^{*}$ equal $M(G)_{U} \cap M(G)_{1}$, relative to the natural involution. In $[8,4.8]$ we showed that the convex hull of $S^{*}=\left\{\xi \delta_{u}: \xi \in \mathbb{T}, u \in G\right\}$ is $S\left(M(G), L^{1}(G)\right)$-dense in $M(G)_{1}$.

Let $\left.{ }^{\#}\right)$ be any isometric involution on $L^{1}(G)$ other than the natural involution, and let $S$ equal $M(G)_{U} \cap M(G)_{1}$, where $M(G)_{U}$ is relative to the involution on $M(G)$ induced from ( $\left.{ }^{\#}\right)$. It suffices to show that the linear span of $S$ is not $S\left(M(G), L^{1}(G)\right)$-dense in $M(G)$. The elements of $S$ are extreme points of $M(G)_{1}$. By [9] the set of extreme points of $M(G)_{1}$ is $S^{*}$. Lemma 1.5 implies that $\xi \delta_{u} *\left(\xi \delta_{u}\right)^{\#}$ equals $\chi(u) \delta_{u^{*} \sigma\left(u^{-1}\right)}$. Thus $\xi \delta_{u}$ is an element of $S$ if and only if $\chi(u)=1$ and $u=\sigma(u)$.

Let $N=\{u \in G: \chi(u)=1$ and $u=\sigma(u)\}$, so $N$ is a closed proper subset of $G$. By the above paragraph, $S$ is contained in $M(N)$. Hence the weak-* closure of the linear span of $S$ is contained in $M(N) \neq M(G)$. Since McKennon in [7] showed on norm bounded sets of $M(G)$ the $S\left(M(G), L^{1}(G)\right)$-topology is finer than the weak-* topology, this completes the proof.

Example 1.6. If $G$ is abelian, then $\sigma(u)=u^{-1}$ is a homeomorphic period two automorphism and every character, $\chi$, satisfies $\chi \circ \sigma=\chi^{-}$. Thus every $\chi \in G^{\wedge}$ defines an involution, $f^{*, \chi}(u)=\chi(u) f(u)^{-}$.

Example 1.7. On any group there is a one-to-one correspondence between nontrivial real characters and open normal subgroups of index two. Any real character and the identity automorphism satisfy the hypotheses of Theorem 1.2, thus every open normal subgroup of index two defines an involution.

Example 1.8. For $\mathbb{R}$ with its usual additive structure and topology there are only two automorphisms of period two, $u \mapsto u$ and $u \mapsto u^{-1}$, and no proper open normal subgroups. Thus the only involutions on $L^{1}(\mathbb{R})$ are the natural involution and $f^{\#, t}(u)=\mathrm{e}^{i t u} f(u)^{-}$for $u, t \in \mathbb{R}, f \in L^{1}(\mathbb{R})$. 


\section{REPRESENTATION THEORY}

Throughout this section $G$ will denote a locally compact group and $\left(^{*}\right)$ will denote the natural involution on $L^{1}(G)$. Using the one-to-one correspondence between nondegenerate ${ }^{*}$-representations of $\left(L^{1}(G),{ }^{*}\right)$ and continuous unitary representations of $G[1,13.3 .1,13.3 .4]$, we are able to prove the following theorem.

Proposition 2.1. Let $\left(^{\#}\right)$ be an unnatural involution on $L^{1}(G)$. Let $H$ be a Hilbert space. If $T$ is a nondegenerate $\left(^{\#}\right)$-representation of $\left(L^{1}(G),{ }^{\#}\right)$ on $H$, then $T$ is also a nondegenerate *-representation of $\left(L^{1}(G),{ }^{*}\right)$ on $H$. Hence $T_{f^{*}}=T_{f^{*}}$ for all $f \in L^{1}(G)$.

Proof. In the proof of $[1,13.3 .4]$ the only ${ }^{*}$-structure used is that a nondegenerate *-representation of $L^{1}(G)$ generates a dense subspace of $H$. This is also true of $T$; hence there is a unitary representation of $G$ that $T$ induces. This unitary representation of $G$ generates a nondegenerate *-representation of $L^{1}(G)$, which the second half of the proof of $[1,13.3 .4]$ shows is equal to $T$.

Since there is a one-to-one correspondence between continuous positive definite functions on $G$ and positive functionals on $\left(L^{1}(G),{ }^{*}\right)[4,32.36]$, one would expect that if $(\#)$ is an unnatural involution, then there is a similar correspondence between a subset of the continuous positive definite functions and positive functionals on $\left(L^{1}(G),{ }^{\#}\right)$. In stating the correspondence the following definition will be useful.

Definition 2.2. Let $\chi$ and $\sigma$ satisfy conditions (1) and (2) of Theorem 1.2. Let $\varphi$ be a positive definite function on $G ; \varphi$ is called a $(\chi, \sigma)$-positive definite function if for any $\alpha_{1}, \ldots, \alpha_{n} \in \mathbb{C}$ and $u_{1}, \ldots, u_{n} \in G$,

$$
\sum_{i, j=1}^{n} \alpha_{i} \bar{\alpha}_{j} \chi\left(u_{j}\right) \varphi\left(\sigma\left(u_{j}^{-1}\right) u_{i}\right) \geq 0 .
$$

Proposition 2.3. Let $\left(^{\#}\right)$ be an unnatural involution on $L^{1}(G)$, with $\chi$ and $\sigma$ the associated automorphism and character from Theorem 1.2. If $p$ is a positive definite functional on $\left(L^{1}(G),{ }^{\#}\right)$, then there exists a unique continuous $(\chi, \sigma)$ positive definite function, $\varphi$, on $G$ with

$$
p(f)=\int_{G} f(u) \varphi(u) d \lambda(u),
$$

for all $f \in L^{1}(G)$.

Proof. By standard representation theory there exists a cyclic ${ }^{*}$-representation, $T$, of $L^{1}(G)$, with $p(f)=\left\langle T_{f} \xi, \xi\right\rangle$ for all $f \in L^{1}(G)$ [3, 21.24; 4, 32.27, 32.28]. By Theorem 2.1, $T$ is also a *-representation of $L^{1}(G)$. The last statement of Theorem 2.1 implies that $p$ is a positive definite functional on $\left(L^{1}(G),{ }^{*}\right)$. Thus by $[4,32.36]$ there exists a unique continuous positive definite function, $\varphi$, such that (1) holds. To complete the proof we need to show that $\varphi$ is $(\chi, \sigma)$-positive definite. On $M(G)$, with the induced involution from $\left(L^{1}(G), \#\right)$, there is a unique extension of $T[2$, VI 19.11]; denote this extension again by $T$. This implies that $p$ has a unique extension to $\left(M(G),{ }^{*}\right)$ which satisfies $p(\mu)=\int_{G} \varphi(u) d \mu(u)$ for all $\mu \in M(G)$. A calculation using this 
equation, the pointmasses, Lemma 1.4 , and the positivity of $p$ on $\left(M(G),{ }^{\#}\right)$ shows that $\varphi$ is $(\chi, \sigma)$-positive definite.

Proposition 2.4. Let $\left(^{\#}\right)$ be an unnatural involution on $L^{1}(G)$, with $\chi$ and $\sigma$ the associated automorphism and character from Theorem 1.2. If $T$ is $a$ nondegenerate cyclic \#-representation of $\left(L^{1}(G),{ }^{\#}\right)$ and $\varphi$ is the associated $(\chi, \sigma)$-positive definite function, then

$$
\chi(u)=\frac{\varphi\left(u^{-1} v\right)}{\varphi\left(\sigma\left(u^{-1}\right) v\right)} \quad \text { for all } u, v \in G .
$$

Proof. As in the proof of Proposition 2.3, $T$ induces a ${ }^{\#}$-representation on $\left(M(G),{ }^{\#}\right)$ which is also a *-representation of $\left(M(G),{ }^{*}\right)$ and $\varphi(\mu)=\left\langle T_{\mu} \xi, \xi\right\rangle$ for all $\mu \in M(G)$. Thus if $u$ and $v$ are in $G$, we have $\chi(u) \varphi\left(\sigma\left(u^{-1}\right) v\right)=$ $\left\langle T_{\chi(u) \delta_{\sigma\left(u^{-1}\right) v}} \xi, \xi\right\rangle=\left\langle T_{\delta_{u}^{*} * \delta_{v}} \xi, \xi\right\rangle=\left\langle T_{\delta_{u}{ }^{*} * \delta_{v}} \xi, \xi\right\rangle=\varphi\left(u^{-1} v\right)$, which completes the proof.

Using the above proposition we will give an example of an isometric involution, $\left(^{\#}\right)$, on $L^{1}(G)$ such that $\left(L^{1}(G),{ }^{\#}\right)$ is \#-radical. This is in marked contrast to the situation of $L^{1}(G)$ with the natural involution, where $L^{1}(G)$ is *-semisimple.

Example 2.5. Let $G$ be a group that contains a proper normal subgroup of index two. By Example 1.7 there exists an unnatural involution, $\left({ }^{\#}\right)$, defined by the identity automorphism and the real character, $\chi$, associated to the subgroup. In (2) of Proposition 2.4 if we let $v$ equal the identity element of $G$, we see there are no nonzero $\left(\chi, \sigma_{\mathrm{id}}\right)$-positive definite functions and hence no nontrivial nondegenerate \#-representations of $\left(L^{1}(G),{ }^{\#}\right)$. Thus $\left(L^{1}(G),{ }^{\#}\right)$ is a $\left(^{\#}\right)$ radical algebra.

Lastly, if $\left(^{*}\right)$ is an unnatural involution on $L^{1}(G)$, with $\chi$ and $\sigma$ the associated automorphism and character from Thereom 1.2, the relevant object for discussing the relationship between the nondegenerate \#-representations of $\left(L^{1}(G),{ }^{\#}\right)$ and representations of $G$ is what we call a $(\chi, \sigma)$-representation of $G$; i.e., a representation, $V$, of $G$ such that $V_{u}^{*}=\chi(u) V_{\sigma\left(u^{-1}\right)}$ for all $u \in G$. Using Theorem 2.1 and the standard construction of a representation of $M(G)$ from a representation of $G\left(T_{\mu}=\int_{G} V_{u} d \mu(u)[1,13.3 .1]\right)$, one shows there is a one-to-one correspondence between nondegenerate representations of $\left(L^{1}(G),{ }^{\#}\right)$ and continuous unitary $(\chi, \sigma)$-representations of $G$. It is interesting to note that Theorem 2.1 implies any bounded continuous $(\chi, \sigma)$-representation (i.e., $\left.\sup \left\{\left\|V_{u}\right\|: u \in G\right\}<\infty\right)$ is also a unitary representation. The author is unable to find an example of a continuous $(\chi, \sigma)$ representation of some group that is unbounded or alternatively show that all continuous $(\chi, \sigma)$-representations are bounded.

\section{ACKNOWLEDGMENT}

The author would like to thank the referee for the suggestions that simplified the proofs of Theorems 1.3 and 1.5. 


\section{REFERENCES}

1. J. Dixmier, $C^{*}$-algebras, North-Holland, New York, 1982.

2. J. M. G. Fell and R. S. Doran, Representations of *-algebras, locally compact groups, and Banach *-algebraic bundles, Vol. 1, Academic Press, San Diego, 1988.

3. E. Hewitt and K. Ross, Abstract harmonic analysis, Vol. 1, Springer-Verlag, Berlin, 1979.

4. __ Abstract harmonic analysis, Vol. 2, Springer-Verlag, Berlin, 1970.

5. E Hewitt and K. Stromberg, Real and abstract analysis, Springer-Verlag, New York, 1965.

6. B. Johnson, Isometric isomorphisms of measure algebras, Proc. Amer. Math. Soc. 15 (1964), 186-187.

7. K. McKennon, Multipliers and positive functionals, Mem. Amer. Math. Soc., vol. 71, Amer. Math. Soc., Providence, RI, 1971.

8. P. Patterson, Characterizations of algebras arising from locally compact groups, Trans. Amer. Math. Soc. 329 (1992), 489-506.

9. R. Rigelhof, $A$ characterization of $M(G)$, Trans. Amer. Math. Soc. 136 (1969), 373-379.

10. J. G. Wendel, Left centralizers and isomorphisms of group algebras, Pacific J. Math. 2 (1952), 251-261.

Department of Mathematics, SAint Louis University, Saint Louis, Missouri 63103-2097

E-mail address: pattersonplesluvca.slu.edu 